\title{
Survey of Job Satisfaction and Bipolar Disorder among Fitness Instructors of Pune City
}

\author{
Moirangthem Hennary Devi ${ }^{1}$, Gaurav Pant ${ }^{2}$ \\ ${ }^{I}$ MPhil Scholar, Department of Physical Education, Bharati Vidyapeeth Deemed University, Pune, India \\ ${ }^{2}$ Assistant Professor, Department of Physical Education, Bharati Vidyapeeth Deemed University, Pune, India'
}

\begin{abstract}
Background: The concept of job satisfaction has been developed in many ways by many form properly different researchers and practitioners. One of the most widely used definitions in organizational research is that of Locke, who defines job satisfaction as "a pleasurable or positive emotional state resulting from the appraisal of one's job or job experiences". Bipolar disorder, also known as manic-depressive illness, is a brain disorder that causes unusual shifts in mood, energy, activity levels, and the ability to carry out dayto-day tasks.
\end{abstract}

Purpose: The purpose of this study is to assess the status of job satisfaction and bipolar disorder among the fitness instructors of Pune city.

Setting and Design: The subjects taken for this study were 100 females (50 fitness instructors \& 50 spa therapist) from various fitness and spa centre of Pune city, age ranging from 21-28 years. Questionnaire was used as a tool for this survey.

Methods: The current study was performed on the survey of job satisfaction and bipolar disorder among fitness instructors of Pune city and to complete this study questionnaire had been given to 100 subjects; the collected data for the study were assessed on the scoring and norms according to the Job satisfaction questionnaire and Mood disorder questionnaire.

Statistical Techniques: Percentile was applied for this survey of job satisfaction and bipolar disorder among fitness instructors of Pune city.

Result: Gym instructors are more status on job satisfaction than the spa therapists are and as well as more disorder in bipolar status.

Conclusion: Gym instructors are more status on job satisfaction than the spa therapists are and as well as more disorder in bipolar status. Hence, many fitness instructor are eagerly waiting for the job in gymnasium and health centers than the spa therapist.

Key Words: Job satisfaction, Bipolar disorder, Fitness instructors, Spa therapist.

\section{Introduction}

1.1 Background of the study: The concept of job satisfaction has been developed in many ways by many form properly different researchers and practitioners. One of the most widely used definitions in organizational research is that of Locke, who defines job satisfaction as "a pleasurable or positive emotional state resulting from the appraisal of one's job or job experiences". Others have defined it as simply how content an individual is with his or her job; whether he or she likes the job or not. It is assessed at both the global level (whether or not the individual is satisfied with the job overall), or at the facet level (whether or not the individual is satisfied with different aspects of the job).

Bipolar disorder, also known as manic-depressive illness, is a brain disorder that causes unusual shifts in mood, energy, activity levels, and the ability to carry out day-to-day tasks. There are four basic types of bipolar disorder; all of them involve clear changes in mood, energy, and activity levels. These moods range from periods of extremely "up," elated, and energized behavior (known as manic episodes) to very sad, "down," or hopeless periods (known as depressive episodes). Less severe manic periods are known as hypomanic episodes. Bipolar I Disorder - defined by manic episodes that last at least 7 days, or by manic symptoms that are so severe that the person needs immediate hospital care. Usually, depressive episodes occur as well, typically lasting at least 2 weeks. Episodes of depression with mixed features (having depression and manic symptoms at the same time) are also possible. Bipolar II Disorder - defined by a pattern of depressive episodes and hypomanic episodes, but not the full-blown manic episodes described above. Cyclothymic Disorder (also called cyclothymia) - defined by numerous periods of hypomanic symptoms as well numerous periods of depressive symptoms lasting for at least 2 years ( 1 year in children and adolescents).

1.2 The problem and its social relevance: Job satisfaction and bipolar disorder are the psychological variables, which will enhance the development of fitness and health issues. Physical and fitness instructor are very cautious about the health and fitness of their clients to provide a healthy and fit society. Both the variables 
are mental issues, which can provide a great aspect on fitness instructors for their future needs. Therefore, the present investigator has formed the problem entitled, "Survey of job satisfaction and bipolar disorder among fitness instructors of Pune city".

\section{Method}

2.1 Subjects: A total number of 100 female subjects (50 fitness instructors \& 50 spa therapists) were selected randomly from various fitness and spa centre of Pune city and their ages were ranged from 21-28 years.

2.2 Variables of the study: The variables for the present study are as under-

* Job satisfaction.

* Bipolar disorder.

2.3 Tool used: A standard questionnaire for the following variables are:

> Job satisfaction: Dr. (Mrs.) Meera Dixit (2013) "Job Satisfaction Questionnaire" National Psychological Corporation, 4/230, Kacheri Ghat, Agra-282004 (India).

> Bipolar disorder: Hirschfeld et al., (2000) "Development and Validation of a Screening Instrument for Bipolar Spectrum Disorder: The Mood Disorder Questionnaire." American Journal of Psychiatry, volume157 , issue-1, pp.1873-1875.

2.4 Procedure: A total number of 100 female subjects (50 fitness instructors \& 50spa therapists) were selected randomly from various fitness and spa center of Pune city to conduct the test. The test was conducted through the questionnaire on both fitness instructors and spa therapists. They were given the questionnaire separately and thus the data were collected.

\section{Results}

The detailed analysis of the data is presented in this chapter. The data collected from the subject were arranged in a tabular form and to find out the status of the fitness instructors. Percentile was used to find out the percentage value. The entire analysis of the data was done based on the objective of the study. The data was obtained by administrating "job satisfaction questionnaires and bipolar disorder questionnaire" followed by its norms to the subject. The scores were obtained by using the key as suggested by Dr. (Mrs.) Meera Dixit (2013) and Hirschfeld (2000).

3.1 Findings: The statistical results of survey of job satisfaction and bipolar disorder among fitness instructors of Pune city have been presented in tables 1 to 4 .

Table-01: Status of the Job Satisfaction on Spa Therapist in Percentile

\begin{tabular}{|c|c|c|c|c|c|c|c|c|}
\hline \multicolumn{2}{|c|}{} & \multicolumn{7}{|c|}{ Percentiles } \\
\cline { 3 - 9 } & 5 & 10 & 25 & 50 & 75 & 90 & 95 \\
\hline Weighted Average & SPA & 174.65 & 179 & 184 & 201 & 210.25 & 218.6 & 225.35 \\
\hline
\end{tabular}

From the Table- 01, signifies the percentile position of the job satisfaction on spa therapist. The average of the raw data is 199 that is between the $25^{\text {th }}$ and $50^{\text {th }}$ score. The percentage of the raw data lies with $73.56 \%$.

Table-02: Status of the Job Satisfaction on Gym Instructor in Percentile

\begin{tabular}{|l|c|c|c|c|c|c|c|c|}
\hline \multicolumn{2}{|c|}{} & \multicolumn{9}{c|}{ Percentiles } \\
\cline { 3 - 9 } & 5 & 10 & 25 & 50 & 75 & 90 & 95 \\
\hline Weighted Average & GYM & 174.6 & 184 & 196.5 & 201 & 206.25 & 218.7 & 22.9 \\
\hline
\end{tabular}

From the above Table-02, signifies the percentile position of the job satisfaction on gym instructor. The average of the raw data is 200.86 that is between the $25^{\text {th }}$ and $50^{\text {th }}$ score. The percentage of the raw data lies with $73.92 \%$.

Table-03: Status of the Bipolar Disorder on Spa Therapist In Percentile

\begin{tabular}{|c|c|c|c|c|c|c|c|c|}
\hline & & \multicolumn{7}{|c|}{ Percentiles } \\
\hline & & 5 & 10 & 25 & 50 & 75 & 90 & 95 \\
\hline Weighted Average & SPA & 16 & 16.2 & 19 & 22 & 25.25 & 28.9 & 30 \\
\hline
\end{tabular}

From the Table- 03, signifies the percentile position of the bipolar disorder on spa therapist. The average of the raw data is 22.48 that is between the $50^{\text {th }}$ and $75^{\text {th }}$ score. The percentage of the raw data lies with $66.11 \%$. 
Survey of Job Satisfaction and Bipolar Disorder among Fitness Instructors of Pune City

Table-04: Status of the Bipolar Disorder on Gym Instructor in Percentile

\begin{tabular}{|l|c|c|c|c|c|c|c|c|}
\hline \multicolumn{2}{|c|}{} & \multicolumn{7}{|c|}{ Percentiles } \\
\cline { 3 - 9 } & 5 & 10 & 25 & 50 & 75 & 90 & 95 \\
\hline Weighted Average & GYM & 19 & 21 & 21 & 25 & 26 & 29.9 & 30.45 \\
\hline
\end{tabular}

From the above Table-04, signifies the percentile position of the bipolar disorder on gym instructor. The average of the raw data is 24.42 that is between the $25^{\text {th }}$ and $50^{\text {th }}$ score. The percentage of the raw data lies with $71.82 \%$.

OVERALL GRAPHICAL REPRESENTATION OF JOB SATISFACTION AND BIPOLAR DISORDER ON SPA THERAPIST AND GYM INSTRUCTOR

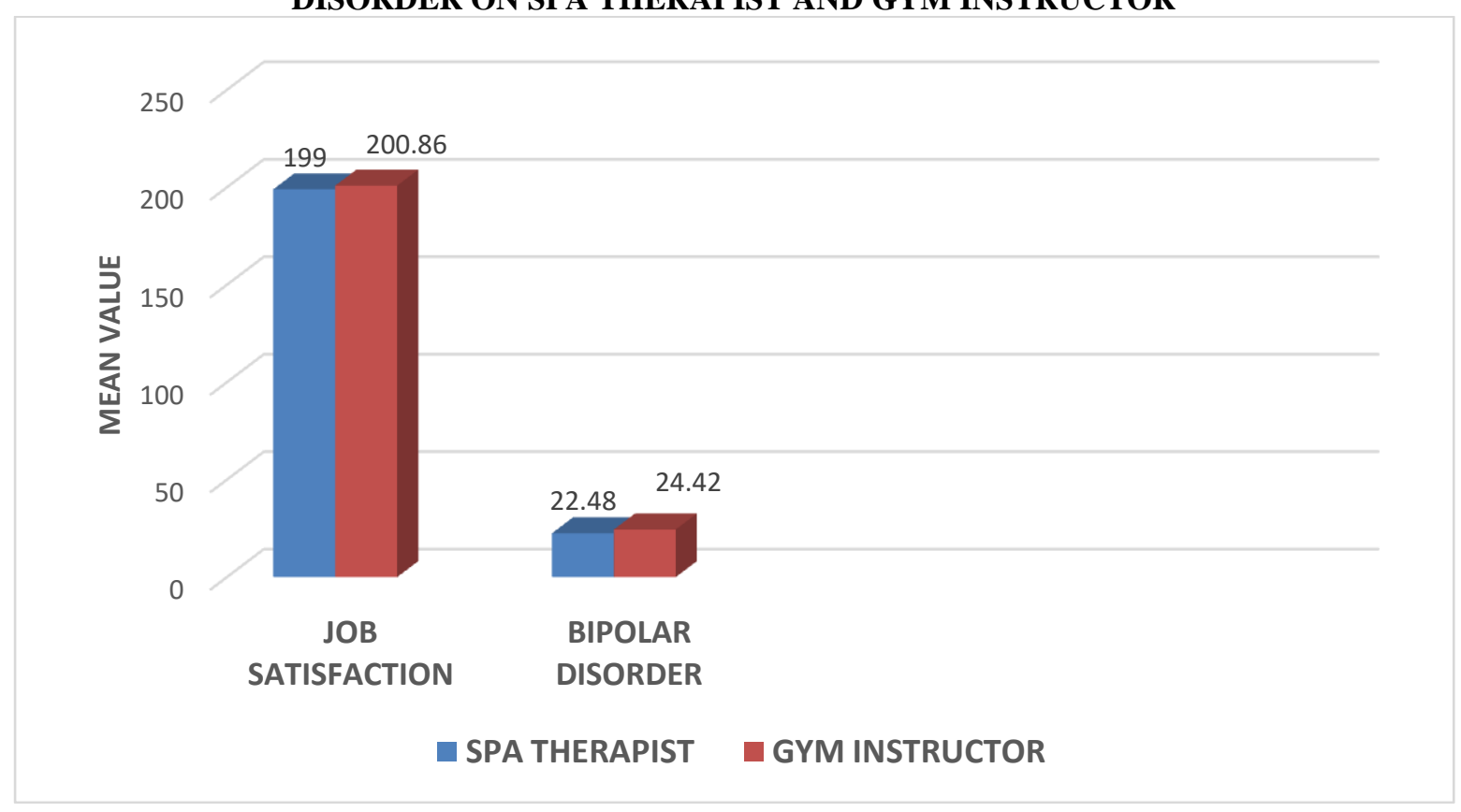

3.2 Discussion of findings: The study reveals that the status of the spa therapist and gym instructor in Pune city on job satisfaction and bipolar disorder. Gym instructor is more status on job satisfaction than the spa therapist is and as well as more disorder in bipolar status. The appearance of this type of results may be due to sports development status and monthly income status of the fitness instructor. Hence, many fitness instructor are eagerly waiting for the job in gymnasium and health centers than the spa therapist.

\section{Conclusion}

On the basis of findings of the present study, the following conclusions are drawn:

$>\quad$ The study reveals that the status of the spa therapist and gym instructor in Pune city on job satisfaction and bipolar disorder. Gym instructor is more status on job satisfaction than the spa therapist is and as well as more disorder in bipolar status.

$>\quad$ In case of job satisfaction, the average percentage are $73.56 \%$ and $73.92 \%$.

$>\quad$ In case of bipolar disorder, $66.11 \%$ and $71.22 \%$ are the percentage that got from the average.

$>\quad$ The appearance of this type of results may be due to sports development status and monthly income status of the fitness instructor. Hence, many fitness instructor are eagerly waiting for the job in gymnasium and health centers than the spa therapist.

According to Di Marco et al., (2016) ${ }^{1}$ conducted a study on "Approaching the Discriminatory Work Environment as Stressor: The Protective Role of Job Satisfaction on Health". Discrimination is a complex phenomenon with adverse consequences at personal and organizational levels. Past studies have demonstrated that workers who are victims of discrimination might show less job satisfaction, less organizational commitment and worse levels of health and productivity. Although most research has focused on the effects of discrimination on victims, less is known about the extent to which discrimination produces consequences on workers who perceive the existence of a discriminatory work environment. The goal of this article is to analyze

${ }^{1}$ Di Marco,(2016). “Approaching the Discriminatory Work Environment as Stressor: The Protective Role of Job Satisfaction on Health". Frontiers in Psychology, volume-07, p.1313. 
the consequences of the perception of a discriminatory work environment on employees' health. The importance of this relationship is studied taking into account the mediating effect of job satisfaction. In order to reach this goal a cross-sectional study was carried out with a sample of 1633 Italian workers $($ male $=826$, female $=764$ ), employed in private and public sectors, and in different hierarchical positions. Results suggest that the perception of a discriminatory work environment is negatively associated with employees' health. This relationship is partially mediated by job satisfaction $(\mathrm{R}(2)=0.17)$. This study demonstrates that perceiving a discriminatory work environment might have a negative impact on workers' health. A higher level of job satisfaction might buffer this effect. These findings have several practical implications. On the one hand, Human Resource Managers need to intervene in order to recognize and diminish implicit biases, creating a healthy and inclusive environment (e.g., through training, diversity policies, etc.). On the other hand, promoting job satisfaction (e.g., providing mechanisms of voice) might help workers to preserve their well-being, coping with the negative effects of a discriminatory work environment.

According to Ashley et al. (2013) examined "A randomized trial of written emotional disclosure interventions in school teachers: con- trolling for positive expectancies and effects on health and job satisfaction". Effects of written emotional disclosure (WED) interventions on the self-reported health and job satisfaction of school teachers, and compared standard WED instructions with two commonly used more prescriptive variants. The study also controlled and measured the between-condition comparability of participants' post-writing benefit expectations. All teachers wrote for $20 \mathrm{~min}$ on three consecutive days at home. Psychological health, physical health, and job satisfaction were assessed at baseline, two weeks, two months, and six-month post-intervention. Participants' expectations of benefit following writing were equivalent across conditions. There was no significant effect of any of the three WED interventions, compared to control writing, on psychological or physical health or job satisfaction. There was, however, a significant and sizeable improvement in physical health across writing conditions from baseline to two-month follow-up, and this was maintained at six months. The findings show that control writing can produce comparable expectations of benefit to WED, and are consistent with the possibility that benefit expectancies can effect health improvements following disclosure or control writing. Most previous studies have examined WED with students or patient groups, and the findings also raise an important question about the feasibility of multi-session writing interventions for mid-life working samples. Further studies with occupational groups are warranted, as is further investigation into the role of positive expectancies in WED effects.

According to Aleisa et al. (2015) measured the "Predictors of global job satisfaction among Saudi physiotherapists: a descriptive study". Level of job satisfaction of PTs working in Saudi Arabia and evaluates predictors of job satisfaction. This was a cross-sectional observational study among licensed physical therapists working across 11 health care centers and university hospitals in Riyadh between 2013 and 2014. A total of 183 physical therapists participated in the survey. Level of job satisfaction and factors influencing satisfaction were explored using a purpose-designed job satisfaction questionnaire. It consisted of 8 survey domains, and the scores were normalized to allow between-domain comparison. Global job satisfaction was $37 \%$. The highest levels of job satisfaction were seen in the domains of professional development and teamwork and the lowest levels of job satisfaction in the domains of supervisory/ management relationship (75\%) and working environment (60\%). Predictors of job satisfaction were gender (OR [odds ratio] 1.4, 95\% CI 1.1-2.3), age (OR 0.7, 95\% CI 0.5-0.9), relationships with supervisors and managers (OR 1.3, 95\% CI 1.2-1.9), working environment (OR 1.2, 95\% CI 1.0-2.3), and opportunities for professional development (OR 1.4, 95\% CI 1.23.7). Saudi PTs were moderately satisfied with their job; strategies should be designed in such a way that they experience a high level of job satisfaction and retention thus resulting in improved rehabilitation services in Saudi Arabia.

[1]. https://en.wikipedia.org/wiki/Job_satisfaction.(12/09/2016).

[2]. https://www.nimh.nih.gov/health/topics/bipolar-disorder/index.shtml (12/09/2016).

[3]. Di Marco, "Approaching the Discriminatory Work Environment as Stressor: The Protective Role of Job Satisfaction on Health". Frontiers in Psychology, 2016, volume-07, p.1313.

[4]. Ashley, (2015) "A randomized trial of written emotional disclosure interventions in school teachers: con- trolling for positive expectancies and effects on health and job satisfaction", Psychological Health Medicine, Volume-18, pp.588-600.

[5]. Aleisa, (2015) "Predictors of global job satisfaction among Saudi physiotherapists: a descriptive study", Ann Saudi Medicine volume- 35 , pp. 46-50. 